\title{
Rational design of viologen redox additives for high-performance supercapacitors with organic electrolytes
}

\author{
Yue $\mathrm{Niu}^{1,2}$, Jin $\mathrm{Niu}^{3}$, Yingjie $\mathrm{Ma}^{1^{*}}$ and Linjie $\mathrm{Zhi}^{{ }^{*}}$
}

\begin{abstract}
Introducing redox species into the electrolytes of traditional electric double layer capacitors (EDLCs) is an efficient strategy to enhance their energy density owing to Faradic reactions. However, few studies have elucidated the effect of the molecular structures of organic redox species on the performance of relative supercapacitors, which is important in the development of redox additives for supercapacitors. In this context, we synthesized several viologens and used them as new organic redox additives for supercapacitors with organic electrolytes. The detailed experimental analysis and theoretical calculation results show that the electrochemical performance of viologens relies heavily on their side chains and conjugated cores. Specifically, the side chains of the viologens affect their electronic structures and are consistent with behaviours between the molecules and the electrode pores due to the size effect, thus influencing their specific capacities. In addition, a larger conjugated aromatic core endows viologens with a smaller band gap and a higher degree of electron delocalization, resulting in better rate performance and cycling stability. Consequently, a $\pi$-conjugated viologen derivative is selected as a favourable additive and enables an EDLC-type supercapacitor to exhibit a high energy density $\left(34.0 \mathrm{~W} \mathrm{~h} \mathrm{~kg}^{-1}\right.$ at $\left.856 \mathrm{~W} \mathrm{~kg}^{-1}\right)$ and good cycling performance.
\end{abstract}

Keywords: organic-soluble viologens, redox additives, molecular design, organic electrolytes, supercapacitors

\section{INTRODUCTION}

In recent years, supercapacitors (SCs) have attracted much more attention as energy storage devices due to their inherent features, such as high power density and excellent cycling stability [1]. However, compared with other energy storage devices, for example, lithium-ion batteries, SCs still exhibit low energy density, which limits their applications. To increase their energy density, many efforts have been made, with a focus on developing various high-performance electrode materials. Very recently, redox-active electrolytes have been reported to be highly promising for improving the performance of SCs because extra capacity can be introduced by Faradaic reactions [2].

Compared with redox electrode materials, redox electrolytes are more convenient for practical applications since they can be simply prepared by directly adding a controlled amount of redox additives into the pure supporting electrolytes. Redox electrolytes can significantly enhance the electrochemical performance of conventional electric double-layer capacitors (EDLCs) with redox additives by introducing Faradic charge storage in addition to EDLC [2]. As a result, investigating high-efficiency redox additives is essential to the production of highperformance redox electrolytes. Until now, many redox additives have been applied to enhance the performance of SCs, such as sulfate salts, iodides and small organic molecules [1,3-8]. Nevertheless, most of the reported studies focus on aqueous electrolyte systems [9-16]. Compared with aqueous systems, organic systems have a broader voltage window that can meet the demands of extensive devices for high voltage and benefit high-energy SCs. In addition, compared with inorganic additives, small organic molecules are more promising additives for

\footnotetext{
${ }^{1}$ CAS Key Laboratory of Nanosystem and Hierarchical Fabrication, CAS Center for Excellence in Nanoscience, National Center for Nanoscience and Technology, Beijing 100190, China

${ }^{2}$ University of Chinese Academy of Sciences, Beijing 100039, China

${ }^{3}$ Key Laboratory of Chemical Resource Engineering, Laboratory of Electrochemical Process and Technology for materials, Beijing University of Chemical Technology, Beijing 100029, China

*Corresponding authors (emails: zhilj@nanoctr.cn (Zhi L); mayj@nanoctr.cn (Ma Y))
} 
organic electrolytes because their solubility and redox potential can be tuned by modifying their chemical structures. However, most of these organic compounds suffer from low power density and poor cycling stability due to their large highest occupied molecular orbital (HOMO)-lowest unoccupied molecular orbital (LUMO) gaps (HLGs) [12,17] and degenerations, respectively. Moreover, little attention has been paid to exploring the effects of the molecular structure of organic additives on their electrochemical performance in SCs, which is, however, critical to maximizing their performance. To further improve the performance of organic electrolytebased SCs, it is necessary to develop new additives with higher redox activity and better stability, and to investigate the effects of their molecular structures on their electrochemical performance.

Among many redox molecules, viologens display reversible two-electron redox activity and thus have been widely selected as active materials in energy storage devices [15,18-20]. For example, using water-soluble viologens as aqueous anolytes along with bromine ions as the oxidized catholytes, improved performance of SCs has been achieved [15]. However, using viologens as active materials in devices results in other problems. For instance, the molecular structures of viologens are closely related to their electrochemical performances [15,21], especially their stability during the redox process, since the side reactions arising from the highly active viologen free radicals during the redox process will result in viologen degenerations, consequently leading to poor stability of SCs. As illustrated by recent work, this issue in aqueous redox flow batteries has been observed and addressed by introducing trimethylammonio groups into viologens, the charge repulsion of which avoids the dimerization of viologen radicals, yielding enhanced cycling stability [21]. In SCs, the structure-property effect of viologens and their degenerations are also critical issues, but few of the corresponding solutions have been reported until now. Consequently, although viologens are promising redox additives for SCs, fabricating viologens for organic electrolytes, which can increase the energy density without greatly sacrificing the power density and cycling stability, is still challenging.

In this work, we demonstrate the design and synthesis of organic-soluble viologens and their superior performance as redox additives for organic electrolytes of traditional EDLCs. By introducing a small amount of these viologens $\left(0.05 \mathrm{~mol} \mathrm{~L}^{-1}\right)$ into pure organic electrolytes, the specific capacity was increased by 2.5 -fold. More importantly, the effects of molecular structures for vio- logens on their electrochemical performance have been investigated to maximize their utilities as redox additives, giving significantly improved specific capacity. Moreover, the HLG of viologens has been successfully decreased by extending the $\pi$-conjugation of viologens, resulting in an obvious enhancement of the electronic transfer kinetics and rate performance. Additionally, extending $\pi$-conjugation efficiently prevents side reactions between viologens. As a result, viologen degeneration during the redox process is significantly reduced, endowing capacitors with long lifetimes as well.

\section{EXPERIMENTAL SECTION}

\section{Materials}

All reagents and solvents were commercially available and used without further purification. 2,4-Dibromothiophene, 4-pyridyl boric acid, potassium carbonate, and tetra(triphenylphosphine palladium) were purchased from Alfa Aesar Company; 4,4-bipyridine, bromoethane, bis-trifluoromethanesulfonimide, and polytetrafluoro-ethylene (PTFE, 60\% dispersed in water) were purchased from Aldrich Company; bistrifluoromethanesulfonimide lithium salt (LiTFSI) was purchased from Shanghai Aladdin Bio-Chem Technology Company; activated carbon (AC) was purchased from Xianfeng Nanotech Company. All solvents were purchased from Beijing Chemical Reagent Company.

\section{Synthesis}

Synthesis of ethyl viologen (EV): to a solution of 4,4bipyridine $(0.83 \mathrm{~g})$ in $\mathrm{N}, \mathrm{N}$-dimethylformamide $(50 \mathrm{~mL})$, $1 \mathrm{~mL}$ bromoethane was added, and the mixture was stirred at $95^{\circ} \mathrm{C}$ for $12 \mathrm{~h}$. The resulting precipitate was collected and washed with dichloromethane (DCM) three times to give a yellow solid. To an aqueous solution of the as-prepared product, excess bis-trifluoromethanesulfonimide $\left(\left(\mathrm{CF}_{3} \mathrm{SO}_{2}\right)_{2} \mathrm{NH}\right)$ was added, and the mixture was stirred at room temperature for $3 \mathrm{~min}$. The resulting precipitate was collected and washed with deionized water three times to give a pale-yellow solid in $95 \%$ yield. ${ }^{1} \mathrm{H}$ NMR (400 MHz, $\mathrm{d}_{6}$-dimethyl sulfoxide) $\delta=9.39$ (d, $J=6.5,4 \mathrm{H}), 8.78(\mathrm{~d}, J=6.4,4 \mathrm{H}), 4.73(\mathrm{q}, J=7.3,4 \mathrm{H}), 1.62(\mathrm{t}$, $J=7.3,6 \mathrm{H}) .{ }^{13} \mathrm{C}$ NMR $(100 \mathrm{MHz}$, acetonitrile) $\delta=150.52$, $146.12,146.03,145.94,127.83,58.37,16.26 .107 .1 . \mathrm{m} / \mathrm{z}$ $[\mathrm{M}-2 \mathrm{TFSI}]^{2+}$ : calculated for $\mathrm{C}_{14} \mathrm{H}_{18} \mathrm{~N}_{2}:$ 107.07; found: 107.07 .

Synthesis of benzyl viologen (BV): to a solution of 4,4bipyridine $(1.0 \mathrm{~g})$ in acetonitrile $(50 \mathrm{~mL}), 1.9 \mathrm{~mL}$ benzyl bromide was added, and the mixture was stirred at $70^{\circ} \mathrm{C}$ 
for $12 \mathrm{~h}$. The resulting precipitate was collected and washed with DCM three times to give a yellow solid. To an aqueous solution of the as-prepared product, excess bistrifluoromethanesulfonimide was added, and the mixture was stirred at room temperature for $3 \mathrm{~min}$. The resulting precipitate was collected and washed with deionized water three times to give a pale-yellow solid in $97 \%$ yield. ${ }^{1} \mathrm{H}$ NMR (400 MHz, $\mathrm{d}_{6}$-acetone) $\delta=9.08(\mathrm{~d}, J=6.6,4 \mathrm{H})$, 8.48 (d, $J=6.4,4 \mathrm{H}), 7.63$ (s, 10H), $5.94(\mathrm{~s}, 4 \mathrm{H}) .{ }^{13} \mathrm{C} \mathrm{NMR}$ (100 MHz, acetonitrile) $\delta=150.96,146.21,133.20$, $130.82,130.29,130.00,129.99,129.99,128.16,65.41 . \mathrm{m} / \mathrm{z}$ $[\mathrm{M}-2 \mathrm{TFSI}]^{2+}$ calculated for $\mathrm{C}_{24} \mathrm{H}_{22} \mathrm{~N}_{2}:$ 169.09; found: 169.09.

Synthesis of $\pi$-extended viologen $(\pi-\mathbf{E V})$ : first, $1 \mathrm{~g}$ of 2,4-dibromothiophene, $1.48 \mathrm{~g}$ of 4 -pyridylboronic acid, $1.66 \mathrm{~g}$ of potassium carbonate and $0.37 \mathrm{~g}$ of tetra(triphenylphosphine palladium) were added into a $250-\mathrm{mL}$ three-port flask. Under the protection of nitrogen, $100 \mathrm{~mL}$ of a 1,4-dioxane and water (4:1) mixture was injected into the flask, and the mixture was stirred under nitrogen at $80^{\circ} \mathrm{C}$ for $72 \mathrm{~h}$. Then, the solvent was evaporated, and the product was separated by chromatography. After evaporation of the solvent, $0.52 \mathrm{~g}$ light yellow powder, 2,5-di(pyridin-4-yl) thiophene, was obtained. To a solution of 2,5-di(pyridin-4-yl) thiophene $(0.20 \mathrm{~g})$ in chloroform $(50 \mathrm{~mL}), 1 \mathrm{~mL}$ bromoethane was added, and the mixture was stirred at $50^{\circ} \mathrm{C}$ for $12 \mathrm{~h}$. The resulting precipitate was collected and washed with DCM three times to give a yellow powder. Excess bis-trifluoromethanesulfonimide was added to an aqueous solution of the as-prepared product, and the mixture was stirred at room temperature for $3 \mathrm{~min}$. The resulting precipitate was collected and washed with deionized water three times to give a white solid in $95 \%$ yield. ${ }^{1} \mathrm{H}$ NMR (400 MHz, $\mathrm{d}_{6}$-dimethyl sulfoxide) $\delta=9.12(\mathrm{~d}, J=6.5,4 \mathrm{H})$, 8.49 (d, $J=6.5,4 \mathrm{H}), 8.47$ (s, 2H), 4.61 (q, J=7.4, 4H), 1.57 $(\mathrm{t}, J=7.2,6 \mathrm{H}) .{ }^{13} \mathrm{C} \mathrm{NMR}(100 \mathrm{MHz}$, acetonitrile $) \delta=$ $148.35,145.19,143.36,133.76,124.36,57.38,16.16 . \mathrm{m} / \mathrm{z}$ $[\mathrm{M}-2 \mathrm{TFSI}]^{2+}$ calculated for $\mathrm{C}_{18} \mathrm{H}_{20} \mathrm{~N}_{2 \mathrm{~S}}$ : 148.07; found: 148.07 .

\section{Cyclic voltammetry}

The cyclic voltammetry (CV) experiments were carried out in a three-electrode cell by a Biologic electrochemical workstation. A glassy carbon disc ( $3 \mathrm{~mm}$ diameter), $\mathrm{Pt}$ wire and $\mathrm{Ag} / \mathrm{Ag}^{+}$served as the working electrode, the counter electrode, and the reference electrode, respectively. The supporting electrolyte was acetonitrile containing $1 \mathrm{~mol} \mathrm{~L}^{-1}$ LiTFSI. Before each test, the glassy carbon disc electrode was polished using polishing alu- mina $(0.05 \mu \mathrm{m})$, and the Pt wire electrode and $\mathrm{Ag} / \mathrm{Ag}^{+}$ electrode were rinsed with water and acetone and then dried by nitrogen.

\section{UV-Vis absorption}

The UV-Vis spectra were obtained on a Shanghai Youke $1810 \mathrm{UV}-\mathrm{V}$ is spectrophotometer. All spectra were recorded under ambient conditions. HLG was calculated according to the equation HLG $=1240 / \lambda_{\text {onset }}$, where $\lambda_{\text {onset }}$ is the onset wavelength of the absorbance peak.

\section{Fabrication of SCs}

Carbon electrode: the electrode was prepared by blending AC (80 wt $\%$, surface area of $2000 \mathrm{~m}^{2} \mathrm{~g}^{-1}$ ), carbon black (15 wt\%) and PTFE (5 wt\%, water dispersion). The mixture became slurry after agitation and was pressed into thin film using a roller. After drying under vacuum at $80^{\circ} \mathrm{C}$ overnight, the electrode film was punched into a circular shape with a diameter of $11 \mathrm{~mm}$ and a mass of approximately $1-1.5 \mathrm{mg}$.

Cell assembly: a two-electrode cell configuration was used to study the SC behaviours with different electrolytes of interest. Two pieces of electrodes with identical mass were pressed in the middle of two stainless steel meshes and used as positive and negative electrodes, respectively. The Whatman glass fibre (GF/D) was cut into circular slices with a diameter of $16 \mathrm{~mm}$ and used as a separator. All capacitors were fabricated in a glove box filled with argon.

\section{Electrochemical methods}

$\mathrm{CV}$ and galvanostatic charge/discharge (GCD) were tested by a Biologic electrochemical workstation at room temperature. The specific capacity $\left(\mathrm{mA} \mathrm{h} \mathrm{g}^{-1}\right)$ of a single electrode was calculated from GCD curves according to the equation: $C=I t / m$, where $I(\mathrm{~A})$ is the constant discharge current, $t(\mathrm{~s})$ is the time for discharging, and $m(\mathrm{~g})$ is the mass of active material in a single electrode. The energy density $\left(E, \mathrm{~W} \mathrm{~h} \mathrm{~kg}{ }^{-1}\right)$ was calculated by $E=$ $(I \oint V \mathrm{~d} t) / 2 m$, where $V$ is the applied voltage.

\section{Brunauer-Emmett-Teller (BET) measurements}

The BET measurement was carried out on ASAP2020, and the density functional theory (DFT) pore model was used to calculate the specific surface area and pore size distribution.

\section{DFT calculations}

DFT calculations were performed using the Gaussian 16 program at the B3LYP/6-311+G(d) level [22]. All energy- 
minimized structures were obtained by vibrational frequency calculations and then used to analyze the HOMO and LUMO.

\section{RESULTS AND DISCUSSION}

As a kind of redox additive for organic electrolytes, the viologens should have efficient redox sites and tunable molecular sizes as well as good solubility in organic solvents. Therefore, as shown in Scheme 1, two organic soluble viologen derivatives (EV and $\mathbf{B V}$ ) were synthesized through a substitution reaction followed by an ionexchange reaction (Scheme S1, Figs S1-S12) $[23,24]$. The two compounds contain different substituent groups, the benzyl and ethyl groups, which are used to adjust the electronic structure of the corresponding viologen molecules and endow them with different sizes to match diverse pores of electrode materials. By introducing TFSI groups, both EV and BV exhibit good solubility in organic solvents, such as acetonitrile and acetone, which enables them to work in organic electrolytes.

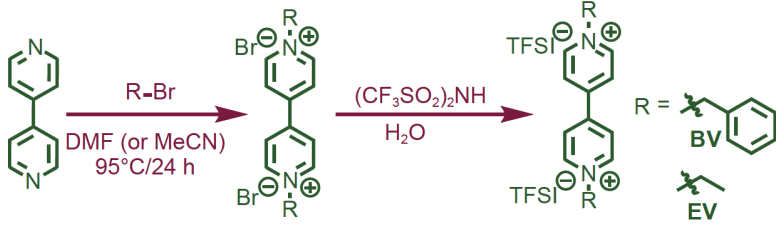

Scheme 1 Synthesis of $\mathbf{E V}$ and $\mathbf{B V}$.

As expected, both $\mathbf{E V}$ and $\mathbf{B V}$ display the typical redox curves of viologens in the CV plot (Fig. S13). In their voltammograms, two anodic peaks as well as two cathodic peaks can be observed, indicating the two-step sequential electron transfer in a reversible process (Fig. 1a, f). The cathodic and anodic peak potentials of $\mathbf{E V}$ and $\mathbf{B V}$ are different (Fig. S13), and BV has a smaller HLG than EV (4.09 vs. $4.18 \mathrm{eV}$ ) according to their UV-Vis spectra (Fig. S14), meaning that different substitution groups affect the redox process of viologens. Because of the electronwithdrawing benzyl groups, BV has a lower LUMO level than EV, resulting in a smaller HLG.

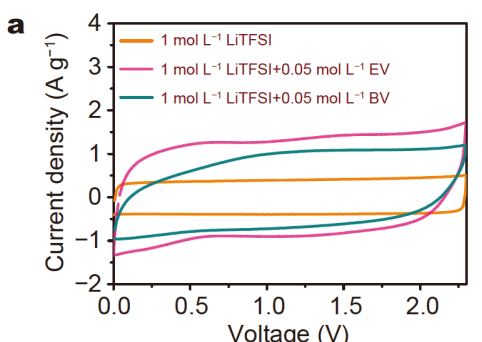

b
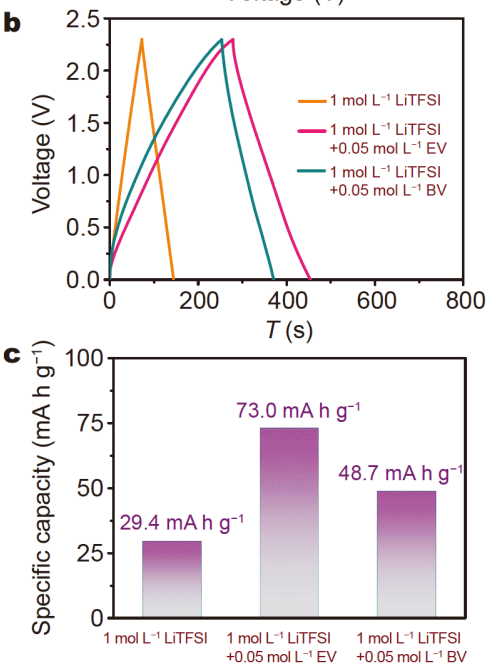
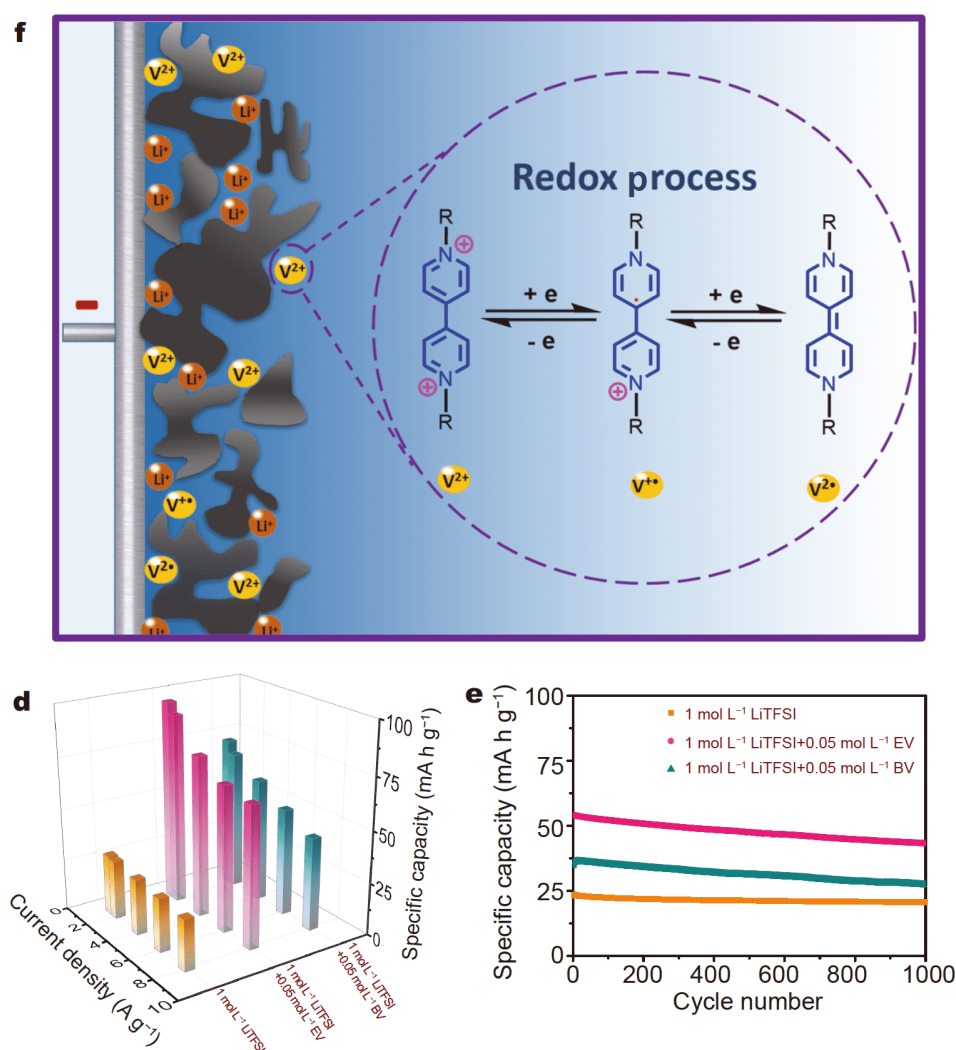

Figure 1 (a) CV curves of the SCs with different electrolytes at a scan rate of $10 \mathrm{mV} \mathrm{s}{ }^{-1}$. (b) GCD curves at different current densities of the SCs with different electrolytes at a current density of $1.5 \mathrm{~A} \mathrm{~g}^{-1}$. (c) Specific capacities of the SCs with different electrolytes at a current density of $1.5 \mathrm{~A} \mathrm{~g}^{-1}$. (d) Specific capacities of the SCs with different electrolytes at varying current densities. (e) Cycling stability of the capacitors with different electrolytes at $4.0 \mathrm{~A} \mathrm{~g}^{-1}$. (f) Redox process of viologen additives on the electrode surface. 
Because of the reversible redox behaviours, $\mathbf{E V}$ and $\mathbf{B V}$ were chosen as redox additives for the organic electrolytes of SCs. EV and BV were added to the bare organic supporting electrolyte ( $1.0 \mathrm{~mol} \mathrm{~L}^{-1}$ LiTFSI in acetonitrile), respectively, obtaining the corresponding redox electrolyte $\left(0.05 \mathrm{~mol} \mathrm{~L}^{-1}\right.$ solution of $\mathbf{E V}$ or $\mathbf{B V}$ in the bare supporting electrolyte). Using these redox electrolytes along with commercial AC as the electrodes, two-electrode cell double-layer capacitors were assembled to evaluate the electrochemical performances of these redox electrolytes. As shown in Fig. 1a, the capacitor with the bare supporting electrolyte shows a rectangular CV curve in the range of 0 to $2.3 \mathrm{~V}$, reflecting that the energy storage of this capacitor is dominated by electric doublelayer capacitive behaviour. Nevertheless, the CV curves of the two capacitors with redox electrolytes, EV and BV, obviously deviate from rectangular due to Faradic reactions of viologen additives on the electrode surface (Fig. 1a, f). Benefiting from the redox behaviours, redox viologens (EV and BV) obviously improved the electrochemical performance of the AC-based EDLCs because of the contribution of the extra capacity from Faradic reactions (Fig. 1a-e). At $1.5 \mathrm{~A} \mathrm{~g}^{-1}, \mathbf{E V}$ and $\mathbf{B V}$ increased the original specific capacity from $29.4 \mathrm{~mA} \mathrm{~h} \mathrm{~g}^{-1}$ to 73.0 and $48.7 \mathrm{~mA} \mathrm{~h} \mathrm{~g}^{-1}$, respectively, which were calculated according to the GCD curves (Fig. 1c). In particular, the specific capacity of the EV-based capacitor is 2.5 times that without redox additive, and the corresponding energy density (calculated based on the weight of active materials) reaches $42.0 \mathrm{~W} \mathrm{~h} \mathrm{~kg}^{-1}$, while the energy density of the capacitor without additive is only $16.9 \mathrm{~W} \mathrm{~h} \mathrm{~kg}^{-1}$.

It should be noted that $\mathbf{B V}$ has a smaller HLG, which should endow it with faster electron transfer kinetics and better rate performance of capacitors. However, the specific capacity of the capacitor with $\mathbf{B V}$ is obviously lower than that of the capacitor with $\mathbf{E V}$ at the same current density. To explore the reason for this unusual phenomenon, the ion conductivities of $\mathbf{B V}$ and $\mathbf{E V}$ containing electrolytes were first measured (Table S1). Compared with the EV-containing electrolyte, the BVcontaining electrolyte has lower ion conductivity (1.74 vs. $1.82 \mathrm{mS} \mathrm{cm}{ }^{-1}$ ), indicating that $\mathbf{B V}$ and $\mathbf{E V}$ affect the rate performance of capacitors by affecting the electrolytes.

In addition, the size effects between the additives and electrode pores were further studied. Based on the theoretical calculation results, the molecular size $(1.548 \mathrm{~nm})$ of $\mathbf{B V}$ is larger than that of $\mathbf{E V}(1.336 \mathrm{~nm})$ due to the benzyl groups (Fig. 2a, b), while most of the pores of electrode materials (AC) have pore sizes below $1.84 \mathrm{~nm}$ (Fig. 2c). Compared with EVs with smaller molecular sizes, fewer BV molecules could insert into the pores of AC due to the stereo-hindrance effect. Since fewer BV molecules are involved in the redox reactions, it shows a lower specific capacity compared with EV (Fig. 2d). Additionally, BV with a slightly larger molecular size might block the channels of electrodes and consequently prevent $\mathrm{Li}^{+}$from going inside the channels, further decreasing the capacity. The rate and cycling performance of the capacitors with $\mathbf{E V}$ and $\mathbf{B V}$ additives were further evaluated. As shown in Fig. 1d, the capacitors with additives show much higher capacities than those without additives, even at high current loads. In addition, the specific capacity of the capacitor with $\mathbf{E V}$ still maintains $\sim 80 \%\left(48.0 \mathrm{~mA} \mathrm{~h} \mathrm{~g}^{-1}\right)$ after 1000 cycles at a current density of $4.0 \mathrm{~A} \mathrm{~g}^{-1}$, which is twice that $\left(23.8 \mathrm{~mA} \mathrm{~h} \mathrm{~g}^{-1}\right)$ of the capacitor without additives (Fig. 1e). Although the capacitor capacity has been obviously improved using BV or $\mathbf{E V}$ additives, the rate and cycling performance of the capacitors still need to be improved when compared with EDLCs.

To further enhance the cycling performance of the capacitors with the viologens, the degeneration of the viologen redox additives should be prevented, which arises from the side reactions of viologen free radicals during the redox process. These radicals formed on the electrode surface during the redox process are highly active and tend to dimerize on the surface (Fig. 3, top), further undergoing disproportionation or other side reactions $[21,25]$. The dimerization is driven mainly by the inherent multicentre covalent $\pi-\pi$ bonding of these radical cation species [26-28]. To inhibit the side reactions during the redox process, extended $\pi$-conjugation was used to tune the molecular structures of the viologens (Fig. 3, bottom). From the molecular perspective, a thiophene unit was introduced into the bipyridine fragment as a linkage between two pyridine rings, obtaining the $\pi$-EV (Scheme S1). The expansion of the conjugation of pyridine units is expected to stabilize viologen radicals by the highly delocalized nature of $\pi-\mathbf{E V}$, preventing side reactions (Fig. 3 ). In the extended $\pi$-conjugated system, the unpaired electron can be delocalized by resonance through effective conjugation, lowering the energy of the molecule and in turn increasing its stability [12,29].

More importantly, the strategy of extended $\pi$-conjugation introduction could simultaneously improve the rate performance of the capacitors with additives. Fast electron transfer kinetics can be achieved by reducing the HLG of the redox molecules through extended conjugation $[12,29]$. To confirm the above deduction, we analyzed the energy levels of $\mathbf{E V}$ and $\boldsymbol{\pi}$-EV at different redox 


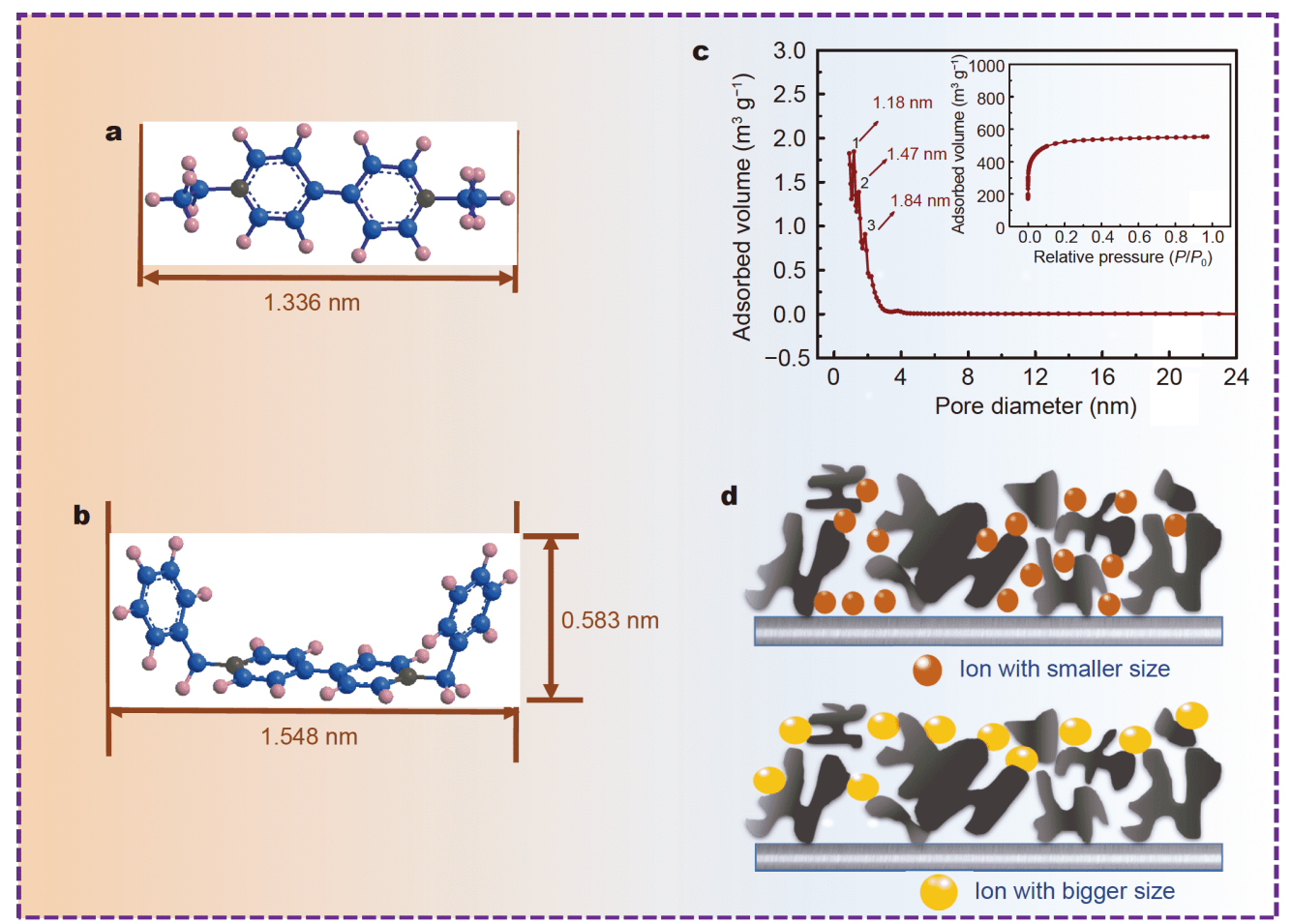

Figure 2 Calculated molecular sizes of (a) EV and (b) BV with lowest energy configuration. (c) Pore size distribution of AC; the inserted diagram shows $\mathrm{N}_{2}$ adsorption-desorption isotherms of AC. (d) Illustration of the match between electrode pores and ions.

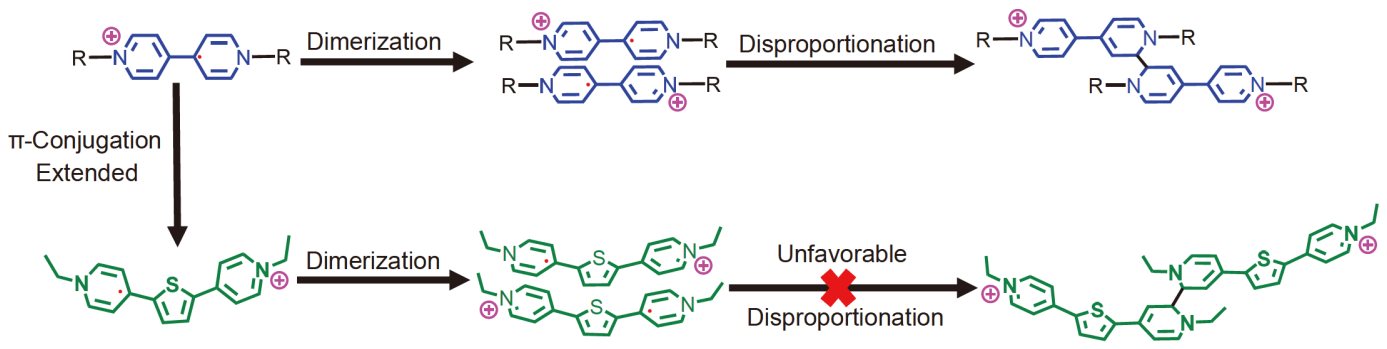

Figure 3 Proposed viologen radical disproportionation of $[\mathbf{E V}]^{+\bullet}$ and unfavourable disproportionation of $[\pi-\mathbf{E V}]^{+\bullet}$.

states by DFT calculations (Fig. 4). Obviously, the HLG of $\pi-\mathbf{E V}$ at each redox state is smaller than that of EV. A smaller HLG gives rise to more reversible redox behaviour and higher electrical conductivity as well, which also benefits the rate performance.

Based on the theoretical optimal design, UV and CV were carried out to investigate the HLG and electrochemical properties of $\pi$-EV experimentally. As shown in Fig. $5 \mathrm{a}$, the maximum UV absorption of $\pi$-EV shows an obvious redshift $\left(\Delta \lambda_{\max }=98 \mathrm{~nm}\right)$ compared with that of EV because of the expansion of $\pi$-conjugation. In addition, $\pi$-EV has a much smaller HLG $(3.01 \mathrm{eV})$ than $\mathbf{E V}$ $(4.18 \mathrm{eV})$, which is consistent with the theoretical calcu- lation result. The electrochemical property of $\pi-\mathbf{E V}$ was evaluated by $\mathrm{CV}$ in an acetonitrile solution of $1 \mathrm{~mol} \mathrm{~L}^{-1}$ LiTFSI (Fig. 5b). $\pi$-EV has two pairs of obvious oxidation-reduction peaks, retaining the typical two electrontransfer process of viologens (Fig. 5c). In particular, compared with $\mathbf{E V}, \boldsymbol{\pi}$-EV shows a smaller difference between the anodic and cathodic peak potentials (Fig. $5 \mathrm{~b})$, named peak-to-peak separation $\left(\Delta E_{\mathrm{p}}\right)$, indicating that $\pi$-EV features more reversible redox behaviour [27]. Electrochemical reversibility is related to the electron transfer kinetics between the electrode and redox additives. Usually, typical electrochemically reversible processes follow the Nernst equation and involve fast 


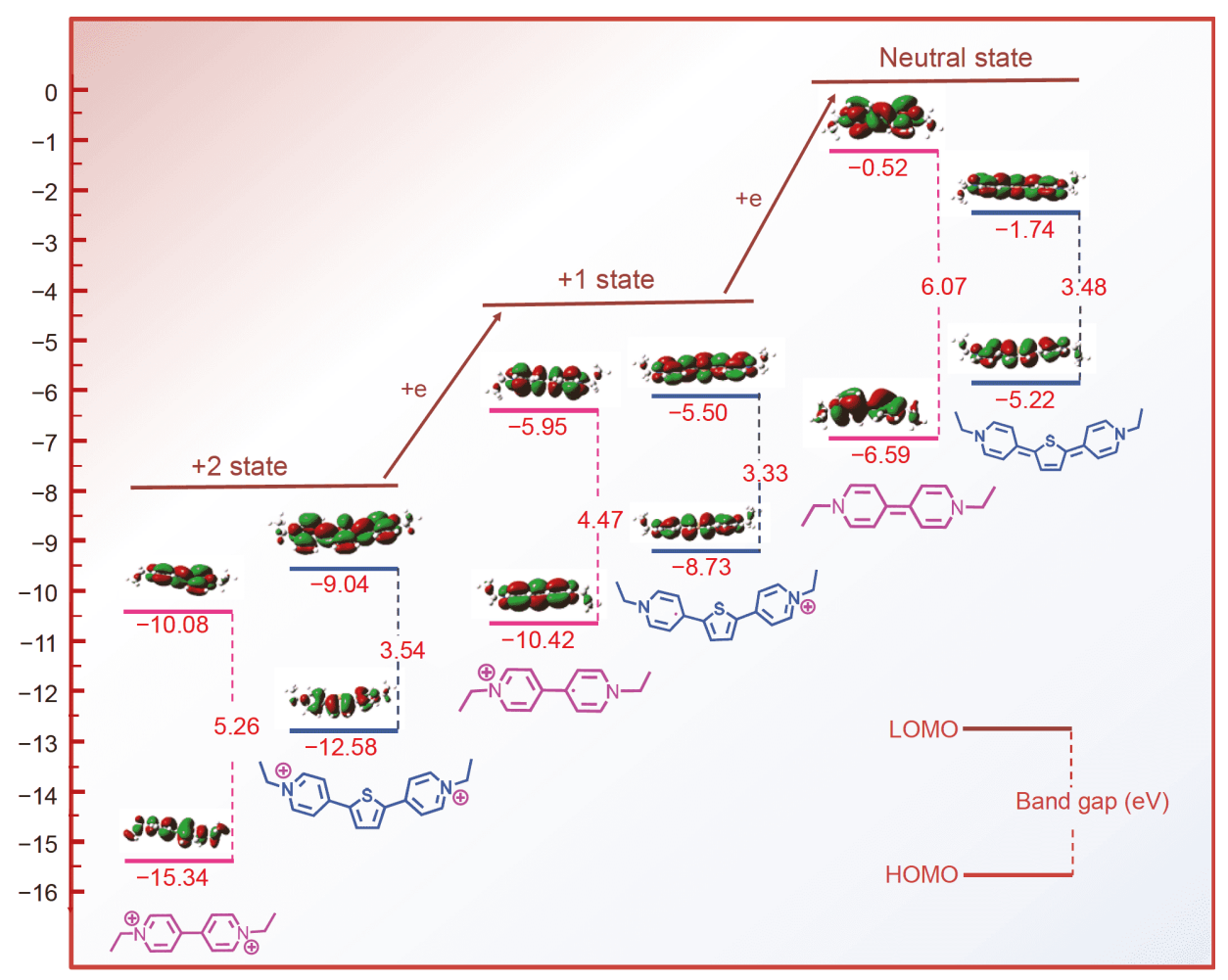

Figure 4 Energy levels of $\mathbf{E V}$ and $\pi$-EV at different redox states by DFT calculation.

electron transfer. However, if there is a high barrier to electron transfer, redox reactions require more negative or positive potentials, and electron transfer processes are delayed, resulting in a larger $\Delta E_{\mathrm{p}}$. In other words, a smaller $\Delta E_{\mathrm{p}}$ of $\pi-\mathbf{E V}$ means that it exhibits faster electron transfer, which is in accordance with the result of HLG measurement. Therefore, as demonstrated in the above optical and electrochemical results, $\pi$-EV molecules with an extended $\pi$-conjugated structure should feature a more efficient charge transfer process and faster redox reaction, thus endowing the capacitor with improved rate performance.

A two-electrode cell was used to evaluate the practical rate and cycling performance of the capacitor with $\pi$-EV additive. The CV curve of the capacitor with redox electrolytes $\pi$-EV also deviates from rectangular due to Faradic reactions (Fig. S15). As shown in Fig. 5d and Fig. S16, the capacitor with $\pi$-EV displays a specific capacity of $59.1 \mathrm{~mA} \mathrm{hg}^{-1}$ at $1.5 \mathrm{Ag}^{-1}$, and the corresponding energy density reaches $34.0 \mathrm{~W} \mathrm{~h} \mathrm{~kg}^{-1}$ at a power density of $856 \mathrm{~W} \mathrm{~kg}^{-1}$. At lower current densities (1.5 to $6.0 \mathrm{~A} \mathrm{~g}^{-1}$ ), the specific capacity of $\pi-\mathbf{E V}$ is lower than that of $\mathbf{E V}$, while the opposite phenomenon is observed at high current densities (in the range 8 to $15 \mathrm{~A} \mathrm{~g}^{-1}$ ), which might also be due to the size matching between redox molecules and pores of electrode materials (AC). When charging and discharging at a relatively low current density (1.5 to $6.0 \mathrm{~A} \mathrm{~g}^{-1}$ ), more $\mathbf{E V}$ molecules can be absorbed by the electrodes and consequently contribute more to the capacity of the device. In contrast, fewer $\pi$ EV molecules participate in the redox process due to their larger molecule size, showing limited performance. When charging and discharging at high current density (8.0 to $\left.15.0 \mathrm{~A} \mathrm{~g}^{-1}\right)$, the time scale of the charging and discharging process is not long enough for the diffusion of EV molecules, making it difficult for $\mathbf{E V}$ molecules to enter the small electrode pores. Thus, the difference in their electrochemical performance mainly depends on their redox properties at high current densities. Compared with EV, $\pi$-EV exhibits more reversible redox behaviour that implies faster electron transfer characteristics, consequently showing a higher specific capacity at high current densities. As the current density increases, the specific capacity of the capacitor with EV additive decreases significantly, while the specific capacity of the capacitor with $\pi$-EV additive decreases slightly. As expected, the $\pi$ EV-based capacitor shows better rate performance with $73.0 \%$ capacity retention even at a high current density of 
a

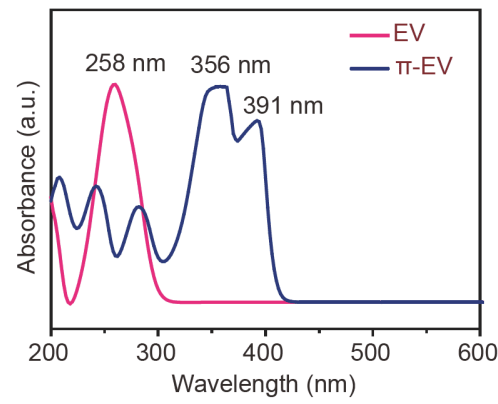

C
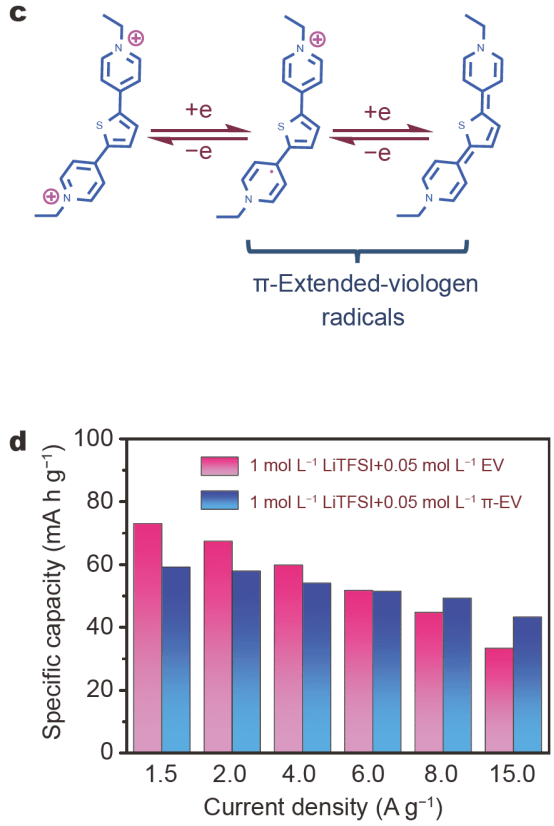
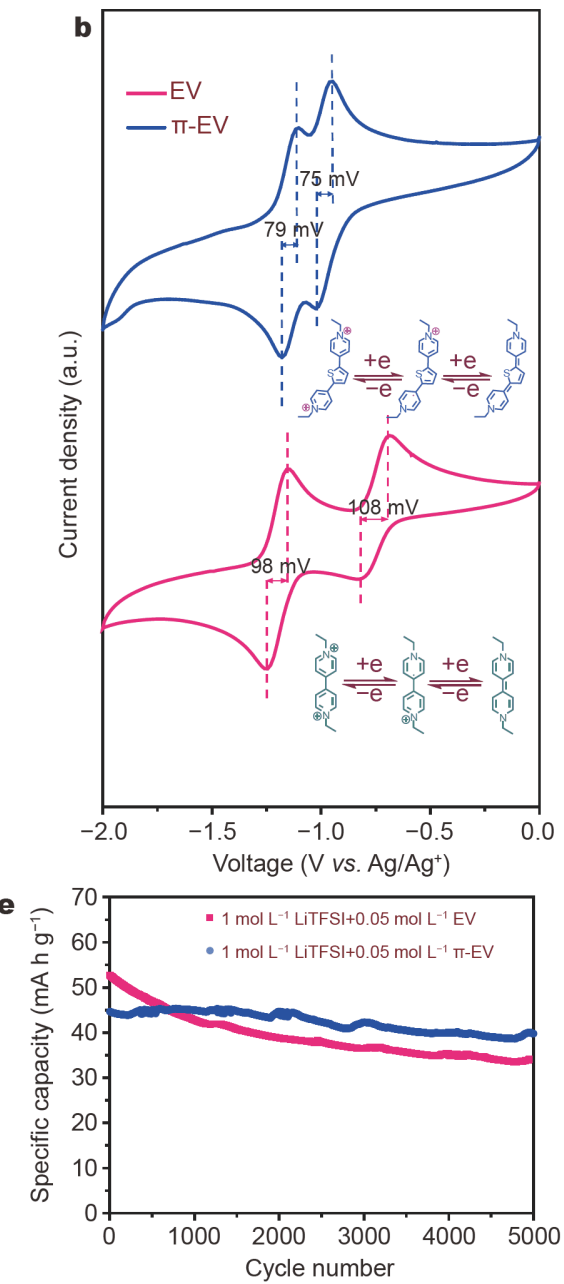

Figure 5 (a) UV absorption of EV and $\pi$-EV in acetonitrile. (b) CV curves of $\pi$-EV in acetonitrile at a scan rate of $100 \mathrm{mV} \mathrm{s}^{-1}$. (c) Reversible process of $\pi$-EV. (d) Specific capacities of capacitors with $\mathbf{E V}$ or $\pi$-EV electrolytes at varying current densities. (e) Cycling stability of capacitors with different electrolytes (EV and $\pi$-EV) at $4.0 \mathrm{~A} \mathrm{~g}^{-1}$.

$15.0 \mathrm{~A} \mathrm{~g}^{-1}$ (compared with the capacity at $1.5 \mathrm{~A} \mathrm{~g}^{-1}$ ), while the EV-based capacitor only has $45.6 \%$ retention at $15.0 \mathrm{~A} \mathrm{~g}^{-1}$. In addition to the improved rate performance, the cyclic performance of $\pi$-EV-based capacitors is also enhanced significantly. After charge-discharge at $4.0 \mathrm{~A} \mathrm{~g}^{-1}$ for 5,000 cycles, the capacitor with $\pi$-EV additive retains $\sim 90 \%$ of the initial specific capacity, while the EV-based capacitor retains $\sim 60 \%$ (Fig. 5e). The enhanced rate and cycling performance of the $\pi$-EV-based capacitor verifies the theoretical and experimental predictions: extended $\pi$-conjugation of $\mathbf{E V}$ endows redox molecules with faster electron transfer kinetics by reducing the HLG and enhances the stability of viologen free radicals due to delocalization of the unpaired electron resonance.

\section{CONCULSIONS}

In summary, a new class of organic-soluble viologen derivatives have been synthesized and successfully used as redox additives in organic electrolytes. The effect of the molecular structures of these redox additives on their electrochemical behaviours in SCs has been clarified, and consequently, improved performance of SCs has been achieved with them. These organic-soluble viologens redox additives dramatically increase the specific capacity of traditional EDLCs via Faradic reactions during charging and discharging processes, achieving remarkable energy density enhancement. Most importantly, the electrochemical performance of viologen additives highly relies on their side chains and aromatic cores. Ethyl group-functionalized viologens fit the pore structures of 
the electrode materials better than those with benzyl groups, exhibiting a higher specific capacity. Notably, the aromatic cores of these viologens are critical to determine their rate performance and electrochemical stability in SCs. Therefore, molecular modification via $\pi$-conjugation extension can significantly improve both the cyclic stability and rate performance of SCs. This work offers distinct insight into the rational design and property modification of electrolyte systems for high-performance SCs and further proposes that the performance of SCs can be tuned rationally by controlling the structure of redox molecules.

\section{Received 21 April 2020; accepted 1 June 2020;} published online 7 August 2020

1 Zhong C, Deng Y, Hu W, et al. A review of electrolyte materials and compositions for electrochemical supercapacitors. Chem Soc Rev, 2015, 44: 7484-7539

2 Evanko B, Boettcher SW, Yoo SJ, et al. Redox-enhanced electrochemical capacitors: status, opportunity, and best practices for performance evaluation. ACS Energy Lett, 2017, 2: 2581-2590

3 Akinwolemiwa B, Peng C, Chen GZ. Redox electrolytes in supercapacitors. J Electrochem Soc, 2015, 162: A5054-A5059

4 Senthilkumar ST, Selvan RK, Melo JS. Redox additive/active electrolytes: a novel approach to enhance the performance of supercapacitors. J Mater Chem A, 2013, 1: 12386

5 Yamazaki S, Ito T, Yamagata $M$, et al. Non-aqueous electrochemical capacitor utilizing electrolytic redox reactions of bromide species in ionic liquid. Electrochim Acta, 2012, 86: 294-297

6 Navalpotro P, Palma J, Anderson M, et al. High performance hybrid supercapacitors by using para-benzoquinone ionic liquid redox electrolyte. J Power Sources, 2016, 306: 711-717

$7 \mathrm{Yu} \mathrm{H}, \mathrm{Wu}$ J, Fan L, et al. An efficient redox-mediated organic electrolyte for high-energy supercapacitor. J Power Sources, 2014, 248: $1123-1126$

8 Sun G, Li K, Sun C. Electrochemical performance of electrochemical capacitors using $\mathrm{Cu}(\mathrm{II})$-containing ionic liquid as the electrolyte. Microporous Mesoporous Mater, 2010, 128: 56-61

9 Fan LQ, Zhong J, Wu JH, et al. Improving the energy density of quasi-solid-state electric double-layer capacitors by introducing redox additives into gel polymer electrolytes. J Mater Chem A, 2014, 2: 9011

10 Lee J, Krüner B, Tolosa A, et al. Tin/vanadium redox electrolyte for battery-like energy storage capacity combined with supercapacitorlike power handling. Energy \& Environmental Science, 2016, 9: 3392-3398

11 Mai LQ, Minhas-Khan A, Tian X, et al. Synergistic interaction between redox-active electrolyte and binder-free functionalized carbon for ultrahigh supercapacitor performance. Nat Commun, 2013, 4: 2923

12 Xiong T, Lee WSV, Chen L, et al. Indole-based conjugated macromolecules as a redox-mediated electrolyte for an ultrahigh power supercapacitor. Energy \& Environmental Science, 2017, 10: 24412449

13 Roldán S, Blanco C, Granda $\mathrm{M}$, et al. Towards a further generation of high-energy carbon-based capacitors by using redox-active electrolytes. Angew Chem Int Ed, 2011, 50: 1699-1701
14 Chun SE, Evanko B, Wang X, et al. Design of aqueous redoxenhanced electrochemical capacitors with high specific energies and slow self-discharge. Nat Commun, 2015, 6: 7818

15 Evanko B, Yoo SJ, Chun SE, et al. Efficient charge storage in dualredox electrochemical capacitors through reversible counterioninduced solid complexation. J Am Chem Soc, 2016, 138: 93739376

16 Yoo SJ, Evanko B, Wang X, et al. Fundamentally addressing bromine storage through reversible solid-state confinement in porous carbon electrodes: design of a high-performance dual-redox electrochemical capacitor. J Am Chem Soc, 2017, 139: 9985-9993

17 Roncali J. Synthetic principles for bandgap control in linear $\pi$ conjugated systems. Chem Rev, 1997, 97: 173-206

18 DeBruler C, Hu B, Moss J, et al. Designer two-electron storage viologen anolyte materials for neutral aqueous organic redox flow batteries. Chem, 2017, 3: 961-978

19 Hagemann T, Winsberg J, Grube M, et al. An aqueous all-organic redox-flow battery employing a (2,2,6,6-tetramethylpiperidin-1-yl) oxyl-containing polymer as catholyte and dimethyl viologen dichloride as anolyte. J Power Sources, 2018, 378: 546-554

20 Liu T, Wei X, Nie Z, et al. A total organic aqueous redox flow battery employing a low cost and sustainable methyl viologen anolyte and 4-HO-TEMPO catholyte. Adv Energy Mater, 2016, 6: 1501449

$21 \mathrm{Hu} \mathrm{B}$, Tang $\mathrm{Y}$, Luo J, et al. Improved radical stability of viologen anolytes in aqueous organic redox flow batteries. Chem Commun, 2018, 54: 6871-6874

22 Frisch MJ, Trucks GW, Schlegel HB, et al. Gaussian 03 Revision B.05. Wallingford: Gaussian Inc, 2004

23 Sathyamoorthi S, Kanagaraj M, Kathiresan M, et al. Ethyl viologen dibromide as a novel dual redox shuttle for supercapacitors. J Mater Chem A, 2016, 4: 4562-4569

24 Takahashi K, Nihira T, Akiyama K, et al. Synthesis and characterization of new conjugation-extended viologens involving a central aromatic linking group. J Chem Soc Chem Commun, 1992, 8: $620-622$

25 Geraskina MR, Dutton AS, Juetten MJ, et al. The viologen cation radical pimer: a case of dispersion-driven bonding. Angew Chem Int Ed, 2017, 56: 9435-9439

26 Zhang DW, Tian J, Chen L, et al. Dimerization of conjugated radical cations: an emerging non-covalent interaction for selfassembly. Chem Asian J, 2015, 10: 56-68

27 Rath H, Tokuji S, Aratani N, et al. A Stable organic radical delocalized on a highly twisted $\pi$ system formed upon palladium metalation of a Möbius aromatic hexaphyrin. Angew Chem Int Ed, 2010, 49: 1489-1491

28 Fahrenbach AC, Barnes JC, Lanfranchi DA, et al. Solution-phase mechanistic study and solid-state structure of a tris(bipyridinium radical cation) inclusion complex. J Am Chem Soc, 2012, 134: 3061-3072

29 Perepichka DF, Bryce MR. Molecules with exceptionally small HOMO-LUMO gaps. Angew Chem Int Ed, 2005, 44: 5370-5373

Acknowledgements We gratefully acknowledge funding support from the Ministry of Science and Technology of China (2012CB933403), Beijing Natural Science Foundation (2182086) and the National Natural Science Foundation of China (51425302 and 51302045).

Author contributions The authors declare that they have no conflict of interest. 
Conflict of interest Zhi L and Ma Y conceived and supervised this work. Niu Y and Ma Y designed the experiments; Niu Y performed the experiments; Niu J carried out the theoretical calculation; Niu Y, Ma Y and Zhi L wrote the paper. All authors revised the paper and contributed to general discussion.

Supplementary information online version of the paper.

Supporting data are available in the

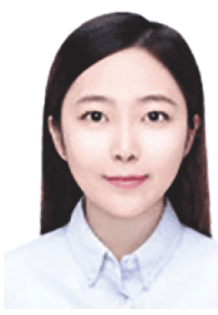

Yue Niu has received her master degree from the National Center for Nanoscience and Technology under the supervision of Professor Linjie Zhi. Now she is pursuing her $\mathrm{PhD}$ degree at the $\mathrm{Na}$ tional Center for Nanoscience and Technology. Her current interests focus on the design and synthesis of organic redox molecules, and their applications in electrochemistry and energy storage.

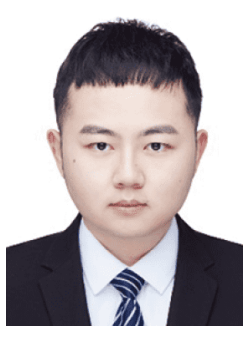

Jin Niu received his $\mathrm{PhD}$ degree from Beijing University of Chemical Technology under the supervision of Prof. Feng Wang in 2018. Then, he joined Prof. Kiyoshi Kanamura's group as a research assistant professor in Tokyo Metropolitan University. Now he is an associate professor in Beijing University of Chemical Technology. His research interests mainly focus on the synthesis and energy applications of $2 \mathrm{D}$ materials and porous materials.

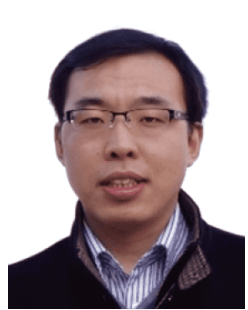

Yingjie Ma received his $\mathrm{PhD}$ in chemistry from Zhejiang University in 2013. Then, he joined Prof. Klaus Müllen's group at Max-Planck Institute for Polymer Research as postdoctor (20132016). Since the end of 2016, he has been an assistant researcher in the National Center for Nanoscience and Technology, China. His research interests focus on syntheses of functional organic molecules and their applications in energy storage and catalysis.

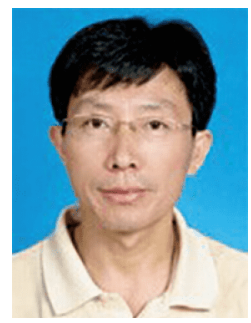

Linjie Zhi received his $\mathrm{PhD}$ in 2000 at the $\mathrm{In}$ stitute of Coal Chemistry, Chinese Academy of Sciences. During 2003-2007, he worked with Prof. Klaus Müllen at the Max-Planck Institute for Polymer Research. Since early 2008, he has been a professor in the National Center for $\mathrm{Na}$ noscience and Technology of China. His research interests focus on carbon-rich nanomaterials and their applications in energy-related areas.

\section{合理设计紫精氧化/还原添加剂分子以构建高性 能有机系超级电容器}

牛越 ${ }^{1,2}$, 牛津 $^{3}$, 马英㷊 ${ }^{1^{*}}$, 智林杰 $^{1^{*}}$

摘要 向双电层电容器的电解液中加入氧化还原活性添加剂是一 种提升其能量密度的有效策略. 添加剂的分子结构与其电化学性 能密切相关, 但目前添加剂“分子结构-电化学性能”的关系还不明 确. 因此, 我们制备了一类适用于有机电解液的紫精基氧化还原添 加剂, 并研究了分子结构对其电化学行为的影响. 研究发现, 紫精分 子的电化学性能与其侧链和共轭骨架密切相关：侧链影响其电子 结构及其与电极孔道之间的匹配, 进而影响其可逆容量; 而更大的 共轭骨架可以赋予紫精分子更小的带隙和更大的电子离域体系, 从而提高其倍率性能和循环稳定性. 结构优化的紫精分子添加剂 可以使双电层电容器获得 $34.0 \mathrm{~W} \mathrm{~h} \mathrm{~kg}$ 的高能量密度和优异的循 环性能. 